\title{
Empirische Validierung von Gestaltungsmustern
}

\author{
Vanessa Petrausch, Christian Schild \\ Heinz Nixdorf Institut, Universität Paderborn
}

\section{Zusammenfassung}

Muster dienen zur Kodifizierung von Gestaltungswissen. Durch eine Einbettung in eine hypothesengeleitete Gestaltung ist es möglich, sie zu Mustersprachen weiterzuentwickeln. Dazu ist es notwendig, die den Mustern zugrunde liegenden Konflikte in Form von Gestaltungshypothesen aufzulösen und empirisch zu validieren, um die weitere Verwendung und Verallgemeinerung zu ermöglichen. In diesem Artikel wird ein Experiment vorgestellt, das dieses Vorgehen beschreibt und anhand zweier Muster exemplarisch das Zusammenspiel zwischen Theoriebildung und Kodifizierung von Gestaltungswissen belegt.

\section{Einleitung}

Mustersprachen und die ihnen zugrunde liegenden Muster haben in der Informatik eine lange Tradition. Seit Beck und Cunningham ihre ersten Ideen zur Nutzung von Mustersprachen in der Informatik skizziert haben (Beck \& Cunningham 1987), wurden zur Entwicklung und Beschreibung von Mustersprachen viele unterschiedliche Wege beschritten. Auf der einen Seite stehen natürlich die von Gamma u. a. entwickelten Muster, die auf der Analyse von bereits existierendem Code basieren (Gamma et al. 1994). Dieses Vorgehen wird auch als Pattern Mining beschrieben (Hanmer \& Kocan 2004). Weiterhin gibt es einige Mustersprachen, die auf Basis von wiederkehrenden Problemen und deren Lösungen möglichst viele bekannte Elemente von Benutzungsoberflächen beschreiben sollen. Hierzu gehören beispielsweise die Muster von Tidwell (Tidwell 2005). Diese Sammlungen von Mustern erfüllen nicht alle Anforderungen, die Alexander (Alexander 1964) an Mustersprachen stellt, da das Konzept der Kräfte nicht berücksichtigt wird. Stattdessen wird versucht, möglichst viele Problemfälle mithilfe der Muster abzuarbeiten. Obwohl dieses Vorgehen von Alexander kritisiert wird, kann diese Kritik vernachlässigt werden, solange das Ziel der Mustersprachen eine Sammlung von tradierten Lösungsmustern ist (Keil \& Schild 2013), ohne sie in eine Mustersprache zu integrieren und weiterzuentwickeln. 
Wie Keil und Schild gezeigt haben, können Muster, die auf den von Alexander beschriebenen Konzepten aufbauen, herangezogen werden, um den Gestaltungsprozess in der Informatik kontextabhängig zu unterstützen (Keil \& Schild 2013). Dazu ist es jedoch notwendig, die gestaltungsrelevanten Kräfte zu identifizieren, die auf die Lösung eines Problems im jeweiligen Kontext einwirken, um eine für diesen Fall optimale Lösung zu finden. Dieses Vorgehen wurde, wie oben beschrieben, bisher in der Informatik eher vernachlässigt.

Darauf folgend geht es in diesem Artikel jetzt darum, für die jeweiligen Lösungen Gestaltungshypothesen aufzustellen und $\mathrm{zu}$ validieren. Auf diese Weise können Mustersprachen entwickelt werden, in denen sich praktische Erfahrungen und theoretische Einsichten verbinden.

\section{Kräfte als gestaltungsleitende Grundlagen}

Nachdem mit den von Keil und Schild vorgeschlagenen Konzepten die ersten Schritte auf dem Weg zu einer, auf der Beschreibung und Lösung von Konflikten basierten, Mustersprache beschritten wurden (Keil \& Schild 2014), soll in diesem Beitrag gezeigt werden, wie sich Muster, die auf solchen Konflikten aufgebaut wurden, empirisch überprüfen lassen. Zunächst müssen dazu die Konflikte identifiziert werden, die den zu erstellenden Mustern zugrunde liegen. Im zweiten Schritt werden dann Hypothesen über die Auswirkungen der jeweiligen Kräfte auf das Verhalten der Benutzer aufgestellt. Die Überprüfung dieser Hypothesen erlaubt es uns anschließend, die Konflikte in ein Muster zu überführen und somit die gewonnenen Erkenntnisse in weiteren Situationen nutzen zu können.

Alexander beschreibt ein Muster wie folgt: »The idea of a diagram, or pattern, is very simple. It is an abstract pattern of physical relationships which resolves a small system of interacting and conflicting forces, and is independent of all other forces, and of all other possible diagrams.« (Alexander 1964, Vorwort) Ausgehend von dieser Beschreibung können wir also die Kräfte als Grundlagen für das Auffinden neuer Muster betrachten. Zu diesem Zweck wurde bereits, wie oben beschrieben, von Keil und Schild eine Systematik vorgestellt, die das Austarieren der Kräfte berücksichtigt und weiterhin als Grundlage für eine hypothesengeleitete Technikgestaltung dienen kann (Keil \& Schild 2014). Um neue Muster zu erstellen, können diese nicht einfach nach Bedarf erfunden, sondern müssen identifiziert werden.

Um neue Muster zu finden und zu validieren, werden hier zwei Probleme identifiziert, die bei der Gestaltung von Benutzungsoberflächen auftreten. Um die Lösungsmöglichkeiten für diese Konflikte zu kodifizieren und so prospektiv in der Gestaltung nutzen zu können, müssen validierte Empfehlungen zur Lösung der Konflikte angegeben werden. So ist es möglich, konfligierende Kräfte und empirische Befunde in einem Muster zu integrieren und in einen Kontext zu setzen. Jetzt geht es darum, mithilfe validierter Gestaltungshypothesen neue Muster aufzuzeigen und in eine Mustersprache zu integrieren. 
Zunächst wird der Konflikt Konsistenz und Lokalität beschrieben. Hierbei wird der Aspekt der generischen Schaltflächen in Dialogboxen thematisiert. In vielen Situationen müssen sich die Entwickler zwischen den beiden Optionen Lokalität und Konsistenz entscheiden, da sich die beiden Anforderungen nicht immer gemeinsam umsetzen lassen. Der zweite Konflikt beschreibt die unterschiedlichen Anforderungen Orts-Konsistenz und Bild-Konsistenz. Das zugrunde liegende Problem in der Gestaltung basiert auf verschiedenen Konsistenzanforderungen, die sich je nach Kontext widersprechen können und je nach Umsetzung die Gedächtnisbelastung für den Benutzer beeinflussen.

\subsection{Konsistenz und Lokalität}

Für die Beschriftung der Schaltflächen in Dialogboxen gibt es zwei Varianten. In der früher gebräuchlichen Variante wurden immer die drei gleichen Schaltflächen eingesetzt (Ja/Yes, Nein/No, Abbrechen/Cancel). Die Schaltflächen wurden also generisch beschriftet und der Beschreibungstext des Dialogs wurde auf die drei möglichen Antworten angepasst. Der Vorteil dieser Variante ist die aus technischer Sicht einfache Wartbarkeit und aus Benutzersicht konsistente Anordnung der Schaltflächen. In der zweiten Variante werden die Schaltflächen direkt mit Handlungsoptionen beschriftet. Diese Variante wird zunehmend häufiger eingesetzt und auch in vielen aktuellen Styleguides empfohlen (Apple 2015, Microsoft 2015 B). An anderer Stelle gibt Microsoft jedoch den generischen Schaltflächen den Vorzug (Microsoft 2015 A).

Die zu prüfende Hypothese beschreibt das folgende Problem. In der ersten Variante (generische Schaltflächen) ist zur Erfassung der Schaltflächenbeschriftung ein geringer Aufwand notwendig. Allerdings ist zur Umwandlung der Antworten Ja, Nein, Abbrechen in die entsprechenden Handlungsoptionen ein zusätzlicher mentaler Aufwand erforderlich. Denn zusätzlich muss mithilfe eines Beschreibungstextes der Nutzer erschließen, welche Konsequenzen jeweils mit den generischen Schaltflächen verknüpft sind, den Beschreibungstext als Frage bzw. mehrere Fragen $\mathrm{zu}$ formulieren, damit die Schaltflächenbeschriftungen Ja, Nein, Abbrechen als Antworten identifiziert werden können. In der zweiten Variante (direkte Handlungsoptionen auf den Schaltflächen) sind der motorische Aufwand zur Erfassung der Schaltflächen höher und die Anordnung nicht immer konsistent, da die Beschriftungen häufig variieren. Jedoch müssen die einzelnen Beschriftungen mental nicht in Handlungsoptionen übersetzt werden, sondern können allein für sich genügend Auskunft über die Konsequenzen der Optionen vermitteln. Der Beschreibungstext der Dialogbox kann dadurch deutlich reduziert werden (Keil 2013, S. 17 ff.). Das bedeutet, dass die Beschreibung direkt am Ort der Handlung untergebracht ist (Lokalität).

Als Hypothese wird davon ausgegangen, dass der mentale Aufwand zur Erschließung der gesamten Dialogbox und der Handlungsoptionen in der ersten Variante (generische Schaltflächen) höher ist als in der zweiten Variante, in der die Schaltflächen direkt mit den Handlungsoptionen beschriftet werden. Daraus lässt sich die Gestaltungsempfehlung ableiten, keine generischen Schaltflächen zu verwenden und die Handlungsoptionen immer direkt auf den Schaltflächen unterzubringen. 


\subsection{Bild-Konsistenz und Orts-Konsistenz}

Auch wenn das Prinzip der Konsistenz häufig als handlungsleitend zur Gestaltung von Benutzungsoberflächen herangezogen wird (Shneiderman \& Plaisant 2004, S. 74), so ergeben sich doch, wie Grudin bereits 1989 schrieb, Probleme, da das Kriterium der Konsistenz nicht einfach zu handhaben ist und häufig Probleme in anderen Bereichen mit sich bringt (Grudin 1989). Beispielsweise kommt es immer wieder zu Problemen zwischen der internen Programmkonsistenz und der Konsistenz der Plattform bzw. des Betriebssystems, da beide Konsistenzen unterschiedliche Ansätze verfolgen. An dieser Stelle sollen nun zwei Arten der Konsistenz und das daraus resultierende Problem betrachtet werden.

Auf der einen Seite steht die Anforderung, dass gleiche Elemente immer am gleichen Ort stehen sollen, da der Benutzer sich diesen Ort für die entsprechenden Handlungen gemerkt hat. Gleichzeitig sollen aber auch für gleiche Aktionen immer die gleichen Piktogramme verwendet werden. Um valide Entscheidungen bezüglich der oben genannten Anforderungen treffen zu können, muss zunächst die Frage beantwortet werden, welche der beiden Anforderungen bei den Benutzern zu einer geringeren Gedächtnisbelastung führt.

In vielen Fällen lassen sich die beiden Anforderungen gleichzeitig vollständig erfüllen. Bei Symbolleisten z. B. tritt dieses Problem in der Regel nicht auf. Jedoch können kurzfristig eingeblendete Handlungsoptionen, wie beispielsweise eine Dialogbox, einen Konflikt hervorrufen. In diesem Fall muss eine Entscheidung getroffen werden, welche der beiden Anforderungen erfüllt werden soll, um eine ergonomische Benutzerführung möglichst wenig zu beeinträchtigen.

Die Hypothese, die in den folgenden Untersuchungen überprüft wird, sagt aus, dass Benutzer sich eher den Ort als das Bild merken. Für die Gestaltung von Benutzungsoberflächen bedeutete dies, dass die Konsistenz des Ortes vorrangig vor der Konsistenz des Bildes behandelt werden sollte. Das Aussehen eines Piktogramms sollte beispielsweise dementsprechend eine untergeordnete Rolle bei der Gestaltung spielen.

\section{Empirische Untersuchung}

Zur Überprüfung beider Hypothesen wurde eine empirische Untersuchung mithilfe eines Eyetrackers durchgeführt. Ein Eyetracker eignet sich zur Überprüfung beider Hypothesen, da jeweils vor allem die Sakkadenbewegungen der Augen Aufschluss über den Bearbeitungsaufwand geben. Viele Sakkadenbewegungen weisen dabei auf viel kognitiven Aufwand hin (Goldberg \& Kotval 1999). Als Eyetracker wurde der SMI RED250 verwendet. Dieser schränkt die Teilnehmer in ihrer Mobilität nicht ein, sodass ein natürliches Arbeitsverhalten während des Experiments möglich war und dadurch Inferenzen von dieser Seite ausgeschlossen werden konnten.

Insgesamt nahmen 28 Teilnehmer aus unterschiedlichen Fachbereichen und Altersklassen an der Studie teil. Die Gruppe setzte sich dabei aus 21 Männern und 7 Frauen zusammen. 18 
Teilnehmer hatten ein Alter zwischen 18 und 29, vier waren zwischen 30 und 39, fünf zwischen 40 und 49 und eine Person über 50 Jahre alt. Die Mehrheit der Teilnehmer kam aus dem Bereich der Informatik, jedoch nahmen auch 11 Personen aus anderen Berufsgruppen wie z. B. Steuerfachangestellte, Sekretärinnen oder Chemieingenieure teil.

Das Experiment zur Analyse des Designkonflikts Konsistenz und Lokalität untersuchte unterschiedliche Augenbewegungen bei Dialogen mit generischen Schaltflächen und Schaltflächen, welche mit ihrer Handlungsoption direkt beschriftet waren. Die Teilnehmer wurden hierfür in zwei Gruppen bezüglich ihrer Berufe und Altersklasse aufgeteilt, um die Benutzer mit besseren IT-Kenntnissen zu verteilen. Alle Dialoge beschrieben das Szenario, dass ein Anwender ungesicherte Änderungen in einem Dokument hat und dieses im Begriff ist zu schließen. Die Dialoge waren dabei immer ähnlich wie in Abbildung 1 aufgebaut.
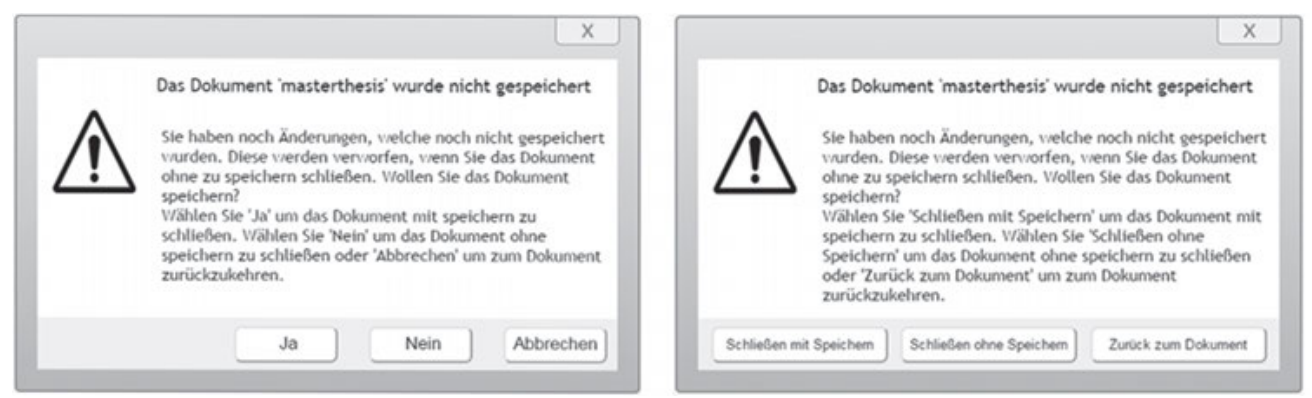

Abbildung 1: Dialogbox mit generischen Schaltflächen und Schaltflächen mit direkten Handlungsoptionen

Der Titel und erste Abschnitt des Dialogs waren in allen Dialogen gleich. In zwei Dialogen wurde zusätzlich zu diesem Abschnitt eine abschließende Frage gestellt. Danach wurden die jeweiligen Schaltflächen erklärt. In zwei Fällen entsprach die Reihenfolge der Erklärung der Anordnung der Schaltflächen entlang der Leserichtung von links nach rechts. In den anderen zwei Fällen war diese Reihenfolge falsch beschrieben. Die Anordnung der Schaltflächen war dabei immer gleich, d. h. die Reihenfolge der Erklärungen im Text war vertauscht. Insgesamt bekamen die Teilnehmer vier verschiedene Dialoge gezeigt. Die Aufgabe war, in jedem Dialog die Option zu wählen, den Dialog mit vorheriger Speicherung der Änderungen zu schließen. Diese konnte in jedem Fall mit einem Klick erreicht werden.

Eine Gruppe hatte nur Dialoge mit Ja/Nein/Abbrechen-Schaltflächen zur Auswahl, die andere Gruppe hatte Schaltflächen, welche mit den jeweiligen Aktionen (Schließen mit Speichern/Schließen ohne Speichern/Zurück zum Dokument) beschriftet waren. Die Dialoge wurden den Teilnehmern nicht direkt nacheinander gezeigt, sondern waren in einem größeren Experiment eingebettet, sodass die Teilnehmer zwischen jedem Dialog ein anderes Experiment durchführen mussten. Dadurch sollte verhindert werden, dass, vor allem im Fall der direkten Beschriftung des Handlungsabschlusses auf den Schaltflächen, die Teilnehmer immer auf die identische Schaltfläche klicken, ohne den Dialog an sich zu beachten. 
Ein weiteres Experiment analysierte den Designkonflikt Bild-Konsistenz und OrtsKonsistenz, welches in Abbildung 2 dargestellt ist. Dazu wurden geometrische Formen in einem regelmäßigen Raster angezeigt. Insgesamt wurden 25 Formen verwendet, wobei einige Formen doppelt vorkommen konnten, jedoch niemals die gesuchte Form. Die Teilnehmer sollten in jedem Durchlauf einen fünfzackigen Stern (Spalte 4, Reihe 2) finden und zur Bestätigung diesen mit der Maus auswählen. Insgesamt wurden 20 Durchläufe in diesem Experiment durchgeführt. Dabei wurde der Stern insgesamt acht Mal gleich positioniert. Vier dieser acht Mal waren dabei innerhalb der ersten fünf Durchläufe, um eine Prägung der Personen auf diese Position zu erreichen. Innerhalb der anderen Positionen wurde der Stern zufällig angeordnet.

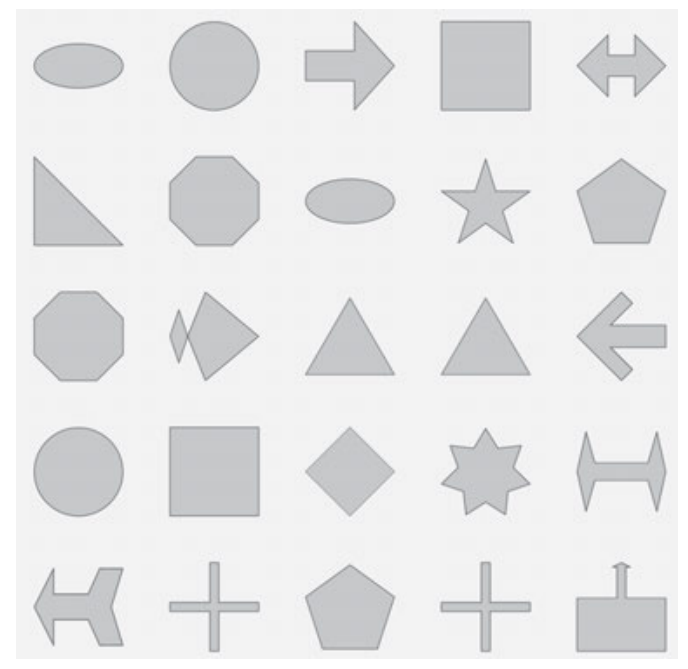

Abbildung 2: Aufbau des Experiments Bild-Konsistenz und Orts-Konsistenz

\section{Ergebnisse}

Abbildung 3 stellt die durchschnittliche Anzahl der Sakkaden für die verschiedenen Iterationen des Experiments für generische und direkt beschriftete Schaltflächen dar. Dabei ist deutlich zu sehen, dass die Anzahl der Sakkaden bei den Dialogen mit generischen Schaltflächen in jedem Durchlauf deutlich höher ist als für Schaltflächen, welche den direkten Handlungsabschluss verdeutlichen. Im Durchschnitt über alle Durchläufe benötigten die Teilnehmer mit den generischen Schaltflächen 78,3 Sakkaden und im anderen Fall 46,63 Sakkaden. Dies entspricht einer Reduzierung von $40 \%$.

Damit kann deutlich gezeigt werden, dass die Augenbewegungen unverkennbar reduziert werden bei der Verwendung von Schaltflächen mit direktem Handlungsabschluss. Eine Analyse der benötigten Zeit für beide Varianten bestätigt dieses Ergebnis. Im Durchschnitt benötigten die Teilnehmer 44 Sekunden, um alle Dialoge mit der richtigen Option im 
generischen Fall zu beenden. Die Teilnehmer des anderen Teils des Experiments benötigten dazu nur 24 Sekunden, sie waren $45 \%$ schneller.

Weiterhin kann mithilfe einer Heat Map aller Teilnehmer gezeigt werden, dass sich der motorische und mentale Aufwand zur Erfassung eines Dialogs deutlich reduziert, wenn die Schaltflächen die Handlungsoptionen direkt anzeigen. Abbildung 4 zeigt eine Heat Map des ersten Dialogs mit generischen Schaltflächen auf der linken Seite und auf der rechten Seite den ersten Dialog mit direkter Beschriftung der Handlungsalternativen. Es ist deutlich zu sehen, dass die Aufmerksamkeitsverteilung im generischen Fall hauptsächlich auf den Text konzentriert ist. Die Teilnehmer waren gezwungen, den Text des Dialogs zu lesen, um die richtige Schaltfläche zur Erledigung der Aufgabe zu finden.

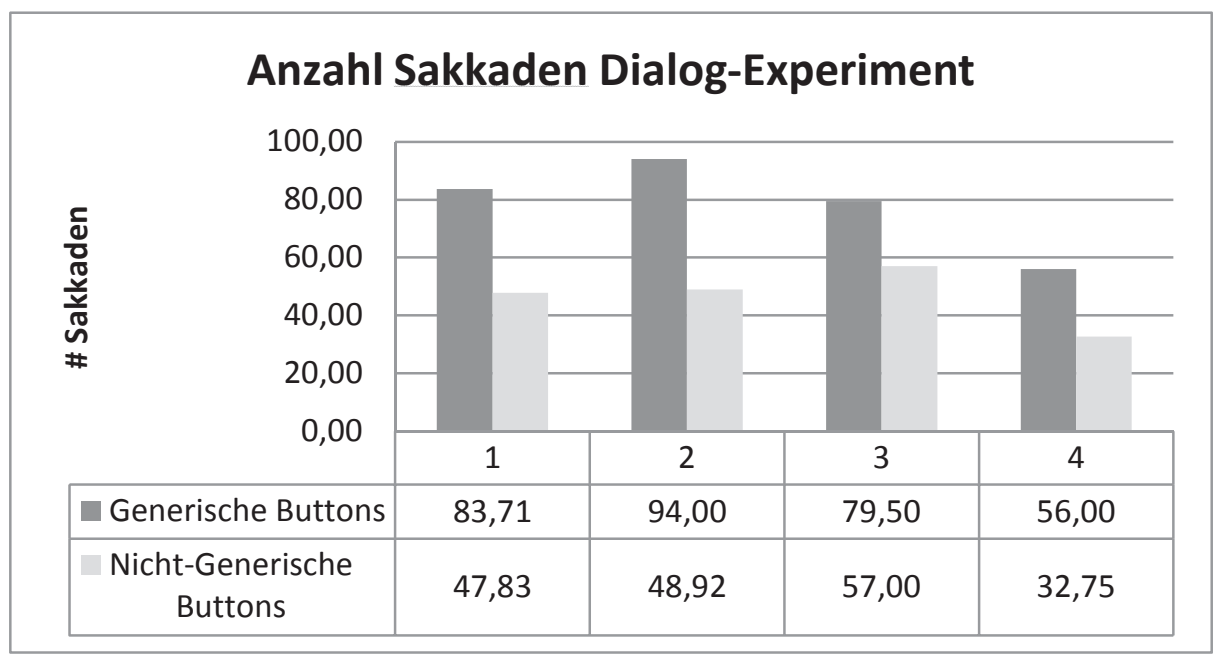

Abbildung 3: Anzahl Sakkaden im Dialog-Experiment

Im Gegensatz dazu zeigt die Heat Map der anderen Version, dass die Aufmerksamkeit der Teilnehmer vermehrt auf den Schaltflächen liegt und der Text im Gegensatz dazu kaum Beachtung findet. Im weiteren Verlauf des Experiments trat dieser Effekt noch stärker auf, sodass im letzten Dialog kaum noch die Aufmerksamkeit auf dem Text lag, sondern nur auf den Schaltflächen. Die generische Version dagegen zeigte immer noch eine Konzentration der Aufmerksamkeit auf den Text. Es konnte entsprechend gezeigt werden, dass durch den Einsatz von Schaltflächen mit direkter Beschriftung der Handlungsoptionen sowohl der mentale als auch der motorische Aufwand reduziert werden. Zusätzlich wurden im generischen Fall zehn Fehler in der Bedienung der Dialoge gemacht, d. h. die Teilnehmer wählten eine falsche Option aus. 90 \% dieser Fehler wurden im zweiten Durchlauf gemacht. Dies könnte die erhöhte Anzahl an Sakkaden in diesem Durchlauf erklären. Im Vergleich dazu wurden im Experiment mit der eindeutigen Beschriftung der Schaltflächen keine Fehler gemacht. 
Die Verwendung von Schaltflächen, welche den direkten Handlungsabschluss verdeutlichen, reduziert somit nicht nur Sakkadenbewegungen und Zeit, sondern führt gleichzeitig zu einer sichereren Bedienung und entlastet folglich den mentalen und motorischen Aufwand bei der Bedienung von Dialogen. Dementsprechend sollten Schaltflächen nicht generisch beschriftet sein, sondern mit den Handlungsoptionen der jeweiligen Schaltflächen. Mithilfe der empirischen Untersuchung konnte der Konflikt Lokalität und Konsistenz zugunsten der Lokalität aufgelöst werden.

Abbildung 5 zeigt die Anzahl der Sakkaden des zweiten Experiments. Die Durchläufe 1, 2, 3, 5, 8, 11, 14 und 17 positionierten den gesuchten Stern immer an der gleichen Stelle. Anhand des Balkendiagramms ist deutlich zu sehen, dass diese Durchläufe jeweils deutlich weniger Sakkaden benötigt haben als andere Durchläufe. Im Durchschnitt benötigten alle Teilnehmer für die Durchläufe mit der geprägten Position 9,31 Sakkaden, für die anderen Durchläufe dagegen 15,02 Sakkaden. Dies entspricht einer Reduzierung von Sakkadenbewegungen um $38 \%$. Eine Analyse der Zeit zeigt, dass die Teilnehmer in den geprägten Durchläufen $17 \%$ weniger Zeit benötigten als in allen anderen Durchläufen. Fehler wurden in diesem Experiment keine gemacht. Eine detailliertere Analyse der randomisierten Positionen zeigte außerdem, dass eine Positionierung des Sterns in den acht benachbarten Positionen der geprägten Position (Durchlauf 9, 12, 18, 19) die Anzahl Sakkaden um $12 \%$ reduziert. Da aufgrund der Randomisierung diese Durchläufe immer nach einem Durchlauf der geprägten Position stattfanden, könnte dieser Effekt stärker auftreten, wenn diese Positionierung nach einem anderen Durchlauf erfolgt wäre. Weitere Experimente könnten darüber Aufschluss geben.
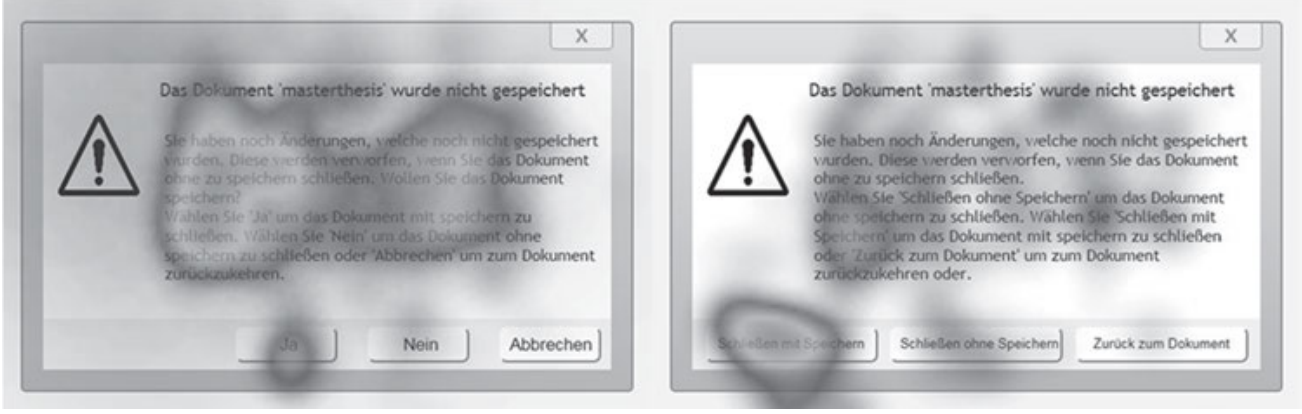

Abbildung 4: Heat Maps der beiden Optionen

Aufgrund der Ergebnisse sollten Schaltflächen und Hinweise daher konsistent bezüglich des Orts und nicht konsistent bezüglich der Bilder gestaltet werden, da dadurch der mentale und motorische Aufwand der Anwender reduziert wird. Der Designkonflikt Bild-Konsistenz und Orts-Konsistenz konnte mithilfe des Experiments in Richtung der Orts-Konsistenz aufgelöst werden. 


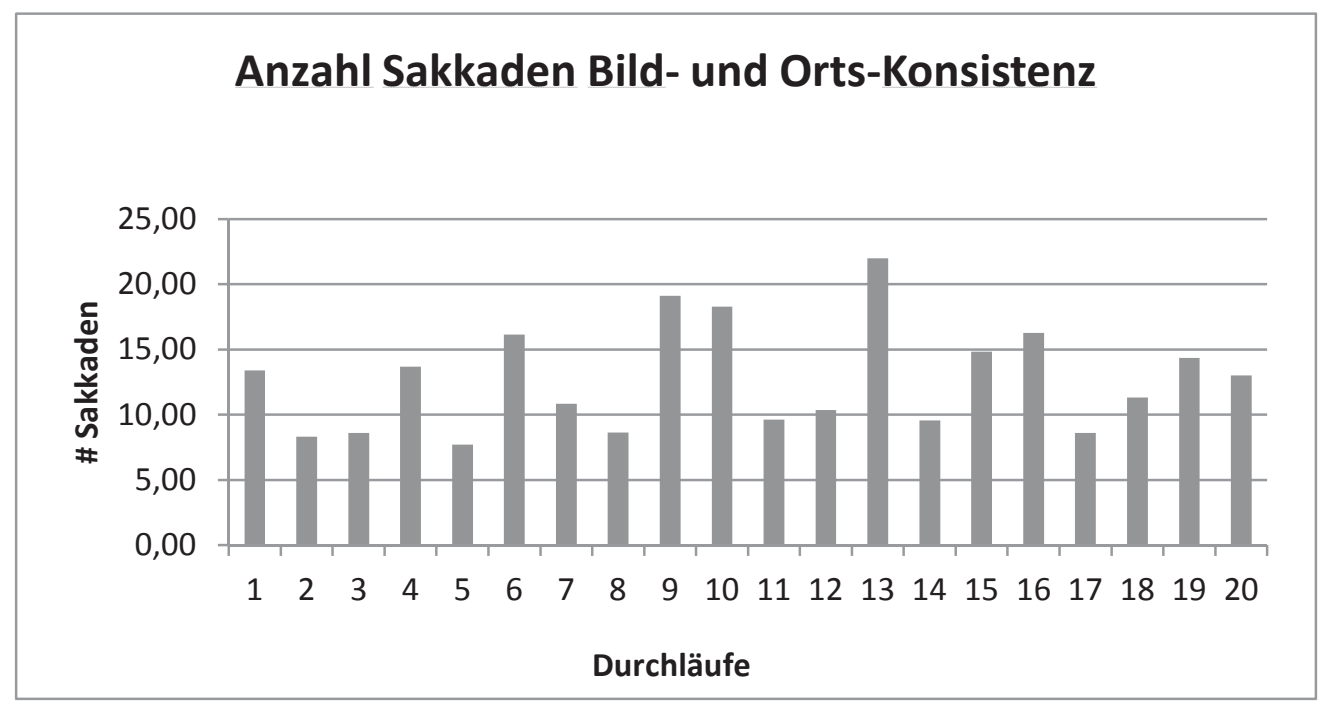

Abbildung 5: Sakkaden im Bild- und Orts-Konsistenz-Experiment

\section{Zusammenfassung und Ausblick}

Mithilfe von zwei Beispielen konnte gezeigt werden, wie sich Muster in der Informatik auffinden lassen und Gestaltungsempfehlungen für die zugrunde liegenden Kräfte aufgestellt und überprüft werden. Im ersten Beispiel wurde eine Gestaltungsempfehlung erstellt, die in der Praxis bereits mehrfach erprobt wurde und so den Ansprüchen von Alexander an Muster entspricht, nachdem die Praxistauglichkeit der Lösungen erwiesen sein sollte. Das zweite Beispiel zeigt Lösungen auf, um die Gedächtnisbelastung für die Benutzer zu minimieren. So konnte mithilfe des Eye-Tracking-Systems eine Gestaltungsempfehlung überprüft und dem Entwickler eine Lösungsmöglichkeit zur Verfügung gestellt werden. Außer den bisher verwendeten Techniken zum Auffinden von Mustern (wissenschaftlich begründete Erkenntnisse) konnte so ein Weg gezeigt werden, bisher häufig verwendete Gestaltungsempfehlungen über eine empirische Untersuchung in eine Software-ErgonomieMustersprache zu überführen. Dazu war es notwendig, die Konflikte und deren Kräfte zu identifizieren. Die anschließende Untersuchung bestätigte die beiden Hypothesen.

Nimmt man hier zusätzlich noch die Erkenntnisse über die Ikonizität von Piktogrammen hinzu (Wandmacher 1993), erhält man eine gute Anleitung, die es Entwicklern gestattet, Designentscheidungen nachvollziehbar $\mathrm{zu}$ treffen und die Entscheidungen auch $\mathrm{zu}$ begründen. Die beschriebenen Muster bilden im Rahmen einer hypothesengeleiteten Technikgestaltung eine Grundlage, um Designwissen zu kodifizieren und zu systematisieren und damit die Weitergabe und -entwicklung dieses Designwissens zu fördern. 


\section{Literaturverzeichnis}

Alexander, C. (1964). Notes on the synthesis of form. Harvard University Press.

Apple (2015). OS X Human Interface Guidelines - Buttons. Website, https://developer.apple.com/library/mac/documentation/

UserExperience/Conceptual/OSXHIGuidelines/ControlsButtons.html. Zuletzt gesichtet 19.3.2015.

Beck, K. \& Cunningham, W. (1987). Using pattern languages for object-oriented programs.

Gamma, E., Johnson, R., Helm, R. \& Vlissides, J. (1994). Design patterns: Elements of reussable object-oriented software. Addison-Wesley.

Goldberg, J. H. \& Kotval, X. P. (1999). Computer interface evaluation using eye movements: methods and constructs. International Journal of Industrial Ergonomics, 24(6) 631-645.

Grudin, J. (1989). The case against user interface consistency. Communications of the ACM, 32(10) 1164-1173.

Hanmer, R. \& Kocan,K. (2004). Documenting architectures with patterns. Bell Labs Technical Journal 9(1) 143-163.

Keil, R. (2013). Technik als Denkzeug: Lerngewebe und Bildungsinfrastrukturen. Wirkungen und Wirksamkeit Neuer Medien in der Bildung, S. 13-30.

Keil, R. (2011). Hypothesengeleitete Technikgestaltung als Grundlage einer kontextuellen Informatik. Breiter, A. \& Wind, M. (Hrsg.), Informationstechnik und ihre Organisationslücken. Soziale, politische und rechtliche Dimensionen aus der Sicht von Wissenschaft und Praxis, S. 165-184.

Keil, R. \& Schild, C. (2013). Gestaltungskonflikte in der Softwareergonomie. Boll, S., Maaß, S. \& Malaka, R. (Hrsg.), Mensch \& Computer 2013 - Tagungsband. Gesellschaft für Informatik. München: Oldenbourg, S. 67-76.

Keil, R \& Schild, C. (2014). Hypothesengeleitete Gestaltung von Benutzungsoberflächen. Butz, A., Koch, M. \& Schlichter, J. (Hrsg.), Mensch \& Computer 2014 - Tagungsband. Gesellschaft für Informatik. Berlin: Oldenbourg, S. 265-274.

Microsoft (2015) A. User interface text. Website, https://msdn. microsoft.com/enus/library/windows/desktop/dn742478.aspx. Zuletzt gesichtet 18.3.2015.

Microsoft (2015) B. User interface principles. Website, https: //msdn.microsoft.com/enus/library/windows/desktop/ff728831(v= vs.85).aspx. Zuletzt gesichtet 18.3.2015.

Shneiderman,B. \& Plaisant, C. (2004). Designing the user interface. Pearson Education.

Tidwell, J. (2005). Designing interfaces - patterns for effective interaction Design: O'Reilly Media, Inc.

Wandmacher, J. (1993). Software-Ergonomie. Bd. 2: Walter de Gruyter.

\section{Kontaktinformationen}

Vanessa Petrausch

Universität Paderborn

v.petrausch@googlemail.com
Christian Schild

Universität Paderborn

christian.schild@uni-paderborn.de 